\title{
Comparison of the photochemical reaction of photoactive yellow protein in crystal with reaction in solution ${ }^{1}$
}

\author{
Eriko Mano, Hironari Kamikubo, Yasushi Imamoto and Mikio Kataoka* \\ Graduate School of Materials Science, Nara Institute of Science and Technology, Ikoma, \\ Nara 630-0101, Japan
}

\begin{abstract}
Photoactive yellow protein (PYP) is a photoreceptor protein for the negative phototaxis of Ectothiorhodospira halophila. The crystal structures of several photo-intermediates have been revealed by X-ray crystallography. In the crystal structure of the active intermediate, $\mathrm{PYP}_{\mathrm{M}}$, no significant structural changes were observed except for the vicinity of the chromophore. On the contrary, spectroscopic studies with solution condition demonstrated that global structural changes occur during the photo-cycle. In order to reveal the origin of the discrepancies, we measured the reaction kinetics upon illumination under crystal condition and to compare them with those observed under solution condition. The reactive portion decreases with the increase of crystallinity. The rate constant of $\mathrm{PYP}_{\mathrm{M}}$ decay also decreases with the increase of crystallinity. These results suggest two possibilities: (1) PYP in crystal does not react by the illumination; (2) the photoreaction rate is highly accelerated in crystal. Consequently, the photoreaction in crystal is considered to be highly influenced by the force constraint from crystalline lattice.
\end{abstract}

\section{Abbreviations}

PYP, photoactive yellow protein from Ectothiorhodospira halophila; MES, 2-(N-morpholino) ethanesulfonic acid; FTIR, Fourier transform infrared, Rg, radius of gyration.

\section{Introduction}

Recent improvements of X-ray crystallographic method have been providing many crystal structures of proteins including their reaction intermediates during their functional expressions. These obtained high-resolution structures give us clues to understand the reaction mechanisms based on their molecular structures. Photoactive yellow protein (PYP) from Ectothiorhodospira halophila is a soluble lightabsorbing protein of $14 \mathrm{kDa}$, and a putative photoreceptor for negative phototaxis [1]. PYP is composed of 125 amino acids and the chromophore, trans-p-coumaric acid [2,3]. The chromophore is isomerized from trans to cis upon absorbing a photon, which triggers a photo-cycle including several photointermediates [4-9]. The crystal structures of dark state and some intermediates have been solved as the results of its useful properties such as solubility, relatively small molecular weight, and the photoreaction triggered by the light illumination.

\footnotetext{
${ }^{1}$ This work was supported in part by a Grant-in-Aid for Scientific Research from the Ministry of Education, Culture, Sports, Science and Technology of Japan.

${ }^{*}$ Corresponding author: Dr. Mikio Kataoka, Department of Materials Science, Nara Institute of Science and Technology, Ikoma, Nara 630-0101, Japan. Tel.: +81 74372 6100; Fax: +81 74372 6109; E-mail: kataoka@ @ms.aist-nara.ac.jp.
} 
The crystal structures of the intermediates formed during the first half of photo-cycle confirmed the isomerization of the chromophore and rearrangements of hydrogen bond network around the chromophore, which are consistent with the knowledge reported by many spectroscopic studies [10]. On the other hand, the crystal structure of $\mathrm{PYP}_{\mathrm{M}}$, proposed to be the active state interacting with the following transducer, shows almost no prominent structural changes without local rearrangements of amino acid residues around the chromophore [11]. Various spectroscopic studies of PYP in solution, however, suggested that the protein portion undergoes global structural changes including a partial protein unfolding [12]. Recently we revealed the structural changes in solution at $\mathrm{PYP}_{\mathrm{M}}$ using small angle solution X-ray scattering [13]. The results show the increase in the radius of gyration $(\mathrm{Rg})$ of about $0.5 \AA$ during $\mathrm{PYP}_{\mathrm{M}}$ formation, whereas the increase in $\mathrm{Rg}$ calculated from the atomic coordinates of crystal structures is negligible small [13].

The discrepancy between the solution structure and the crystal structure of $\mathrm{PYP}_{\mathrm{M}}$ suggest that the structural changes during $\mathrm{PYP}_{\mathrm{M}}$ formation would be influenced by the constraint from the crystalline lattice. The question raised here is what extent the photoreaction is influenced by the restricted structural change due to crystalline lattice. In an attempt to deal with the problem, we measured the decay kinetics of $\mathrm{PYP}_{\mathrm{M}}$ in crystal by time-resolved microspectrophotometry and compared it with that in solution. A single crystal of PYP is too thick to record absorption spectrum. We pressed a single crystal of PYP with a two quartz glasses to obtain poly-crystalline PYP. The method is identical to the previous method applied to measure FTIR spectrum [14]. With the compression, we could measure absorption spectrum and spectral change after light excitation. Photoreaction is observed for solution and the polycrystalline sample with low crystallinity. For the polycrystalline sample with high crystallinity, the spectral change could not be observed with the present time-resolution. This suggests that either the photoreaction does not occur in a crystal or the photoreaction is too fast to observe. The result is an experimental evidence to indicate that the photoreaction of PYP is highly affected by the crystalline fields.

\section{Materials and methods}

\subsection{Sample preparation}

PYP of E. halophila was overexpressed in Escherichia coli, reconstituted by adding p-coumaric anhydride, and purified by DEAE-Sepharose (Amersham Pharmacia Biotech) column chromatography $[15,16]$.

PYP crystals were obtained by vapor diffusion in which $1 \mu$ l of protein solution $(30 \mathrm{mg} / \mathrm{ml})$ was mixed with an equal volume of solution containing 45\% PEG 2000 and 100 mM MES pH 6.5 [17]. A crystal of PYP with the size of $0.5 \times 0.05 \times 0.05 \mathrm{~mm}^{3}$ is normally obtained. The obtained PYP crystal is in a space group $\mathrm{P}_{5}$. The optical density of the crystal is too high to measure the absorbance spectra. In this work, to prepare the suitable thickness to measure the visible spectra, the obtained crystal of PYP was placed on a quartz glass and gently pressed by sealing between the two quartz glasses. The shape and crystallinity of a pressed crystal sample was observed by a polarized microscope (OLYMPUS, SZXILLK100) equipped with polarization filter. The obtained material is expected to be a polycrystalline sample.

\subsection{Time-resolve visible spectroscopy}

The transient visible spectra after photo-excitation were measured by a multi-channel CCD spectroscopy system (S2000 system, Ocean Optics) equipped on the special designed microscope (Tsutsui 
Kougaku). The observed area on the sample is a circle with a diameter of $100 \mu \mathrm{m}$ adjusted by a pin hole placed in front of a receiving optical fiber. PYP was excited by a short arc xenon flash lamp (SA200E, Eagle) for $200 \mu \mathrm{sec}$ with a glass optical filter (Y43, Asahi Techno Glass) which transmits >410-nm light. The deuterium lamp (HAMAMATSU L7893) was used as the monitoring light source. The temperature was kept at $20^{\circ} \mathrm{C}$ during the experiments. The obtained kinetic data were analyzed by IGOR Pro ver.3.14 for Windows (WaveMetrix).

\subsection{FTIR spectroscopy}

The infrared micro-spectroscopy of a single crystal of PYP was carried out using a synchrotron radiation at Beam Line BL43IR in SPring-8 (Japan). A single crystal of PYP was sandwiched between two $\mathrm{CaF}_{2}$ windows, as is the same procedure in the visible spectra measurements, and set in an optical cryostat (MicrostatHe, Oxford Instruments). The infrared beam was focused (20 $\mu \mathrm{m}$ in diameter) on a crystal, and interferograms were averaged. The infrared absorption spectrum of hydrated film of PYP was measured as reported previously [18].

\section{Results}

Figure 1 shows the microscope image of the PYP crystal with and without polarized filters. The two polarizers placed in front and in the rear of the sample are orthogonally set to each other. With this layout, the filters extinguished the background illumination. In fact, we cannot observe the transmitted light outside of the crystal. On the other hand, the part of crystal emits green light. The green light was independent in the orientation between the crystal axis and the direction of the polarization, indicating that green light is not transmitted light through the backward polarizer due to the polarization by the crystal, but luminescence of the crystal. Annealing the crystal at $55^{\circ} \mathrm{C}$ for several minutes dismiss the green light (Fig. 1d). The annealed crystal did not show the X-ray diffraction pattern, indicating the decrease of crystallinity (data not shown). The quenching of the green color with decreasing crystallinity supports the idea that the green light emission can be used as the index of crystallinity.

In order to prepare the sample with adequate thickness to allow the visible spectra measurements, the PYP crystal was sandwitched between the two quartz glasses without spacer and gently pressed, the way

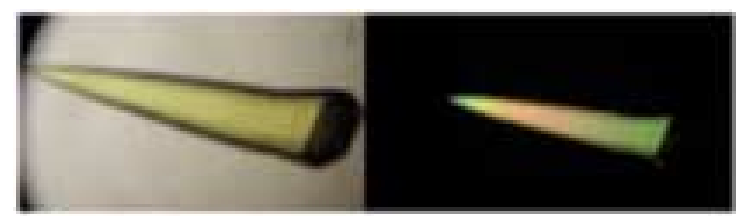

(a)

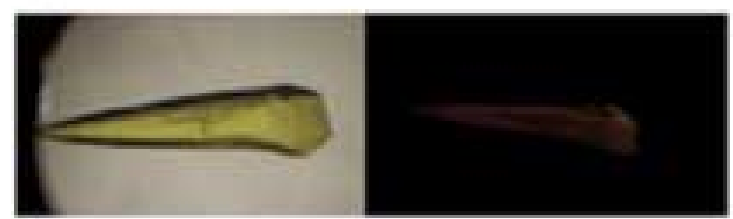

(b)

Fig. 1. The images of optical microscope of PYP crystal. (a) The whole appearance and (b) the polarized image of intact crystal. (c) The whole appearance and (d) the polarized image of annealed crystal at $55^{\circ} \mathrm{C}$. 


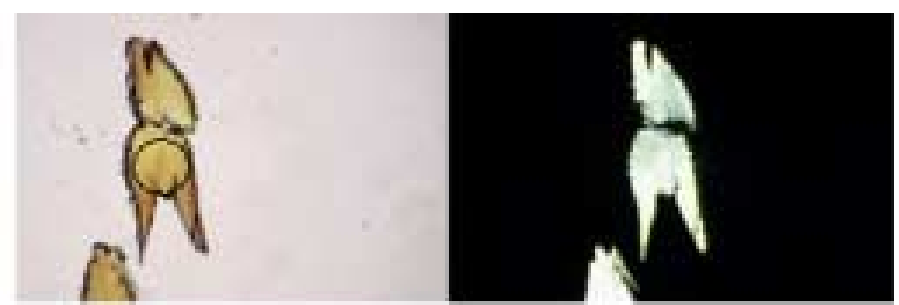

(a)

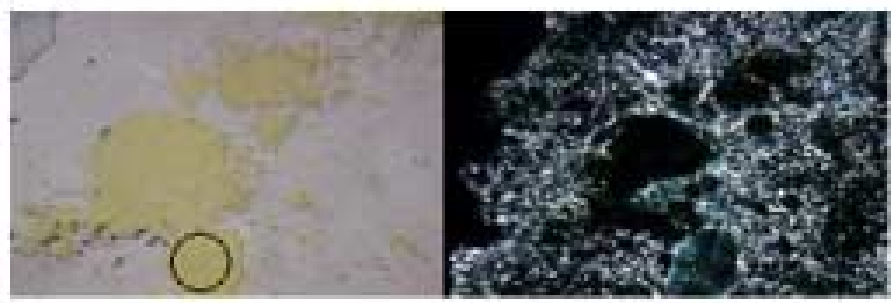

(b)

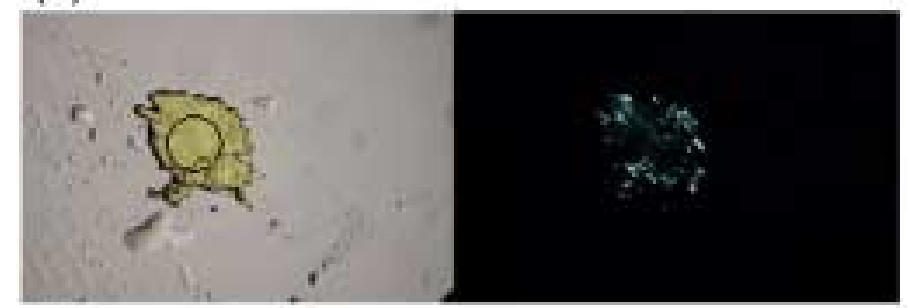

(c)

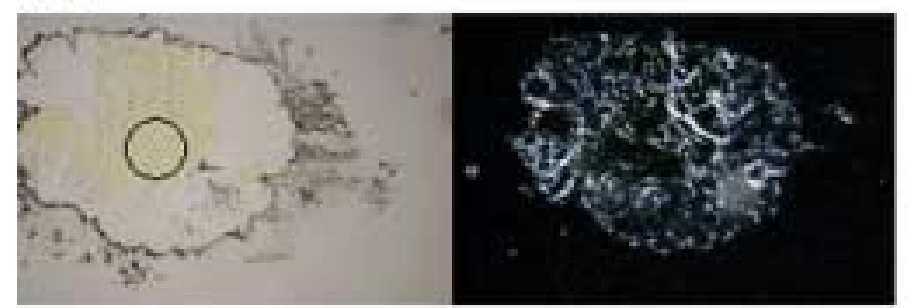

(d)

Fig. 2. The microscopic observations of polycrystalline PYP crystal obtained by gentle pressing. The degree of crystallinity gradually decreases from (a) to (d). Left, the whole appearance; right, polarized image. Circle indicates the region where spectroscopic observation was carried out.

of which has been used in measuring the FTIR spectroscopy of the previous study [18]. Figure 2 shows the microscope images of the crystals independently prepared with the press. The image shows that the prepared sample is polycrystalline PYP. We can easily find the decrease of the bright region in order from top to bottom, suggesting that the region of the crystal with high crystallinity is decreasing in this order. This observation indicates that the crystallinity of the sample is different from preparation to preparation.

The visible absorption spectra were measured for the samples shown in Fig. 2 using the microscope spectrometer. The area where the probed light passed is represented by an open circle in the figure. The diameter of the light is $100 \mu \mathrm{m}$. The visible absorption spectrum of the PYP crystal at $293 \mathrm{~K}$ shown in Fig. $2 \mathrm{~b}$ is given in Fig. 3a. The absorption maximum was located around $445 \mathrm{~nm}$, which is close to that 


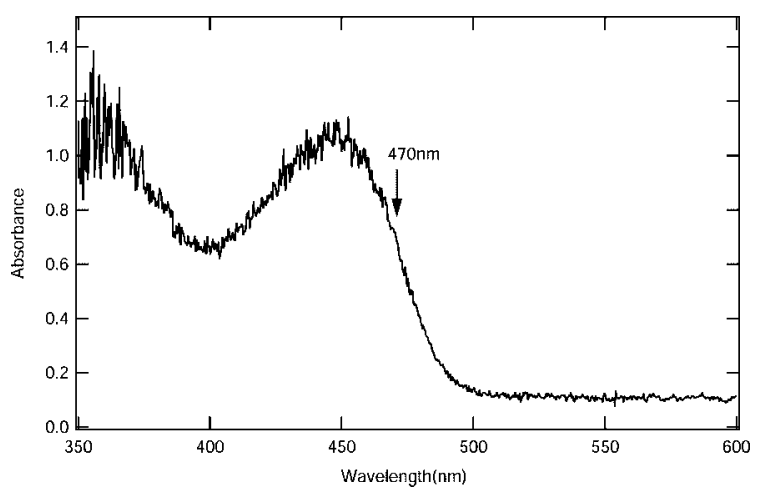

(a)

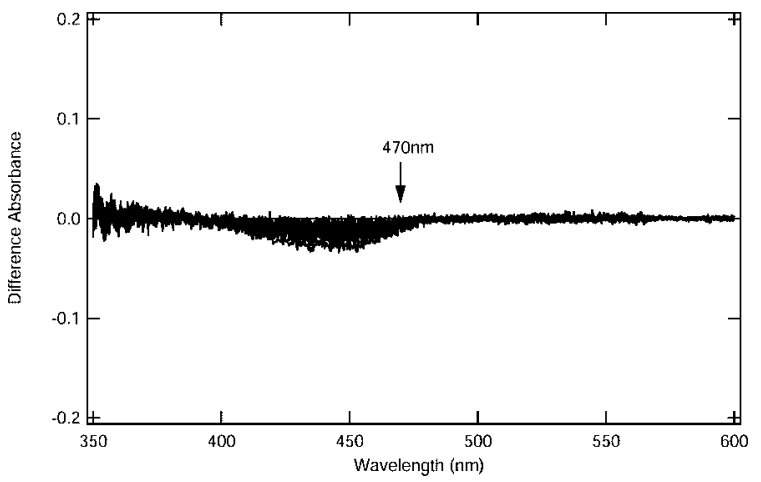

(b)

Fig. 3. Typical microscopic spectrum of polycrystal of PYP. (a) UV-visible absorption spectrum in a dark state. (b) Difference spectral changes after excitation by flash lamp. An arrow indicates the position of $470 \mathrm{~nm}$, where decay kinetics of $\mathrm{M}$ intermediate was observed.

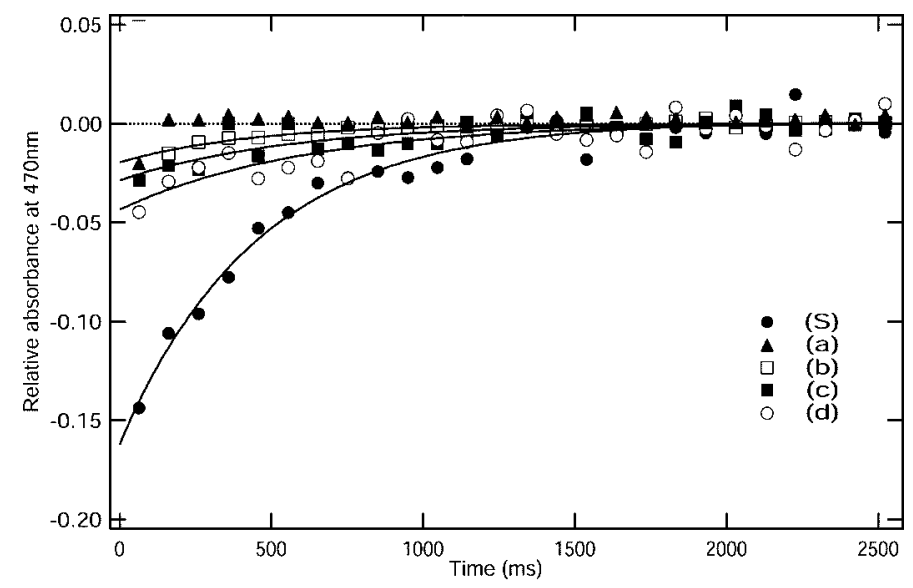

Fig. 4. Relative absorbance changes at $470 \mathrm{~nm}$ for PYP crystal with various crystallinity. The degree of crystallinity decreases in the order of (a), (b), (c), and (d), which are corresponding to Fig. 2. S denotes the kinetics for PYP solution.

in solution condition [19]. The spectra of the other samples also showed the same absorption maximum (not shown), indicating that the visible spectrum was not affected by the crystallinity of the sample. The large noise around $450 \mathrm{~nm}$ is due to the large optical density of the sample. The transient difference absorption spectrum of the sample immediately after the excitation light is shown in Fig. 3b. The base line for Fig. 3b is shown in Fig. 3a. The absorbance around $445 \mathrm{~nm}$ decreases with an isosbestic point at about $400 \mathrm{~nm}$, indicating the accumulation of $\mathrm{PYP}_{\mathrm{M}}[19]$.

Figure 4 shows the decay kinetics of $\mathrm{PYP}_{\mathrm{M}}$ for the samples with various crystallinities. The kinetics was obtained with the absorbance change at $470 \mathrm{~nm}$, although the difference absorption maximum is located at $455 \mathrm{~nm}$. The $\mathrm{S} / \mathrm{N}$ ratio around $455 \mathrm{~nm}$ is considerably high because of the thick concentration due to crystal. The absorbance at $470 \mathrm{~nm}$ is rather accurate than at $455 \mathrm{~nm}$ for quantitative analysis (shown in Fig. 3a,b). The curves were obtained from the samples shown in Fig. 2. The indexes in Fig. 4 correspond to those in Fig. 2. (The curves $a-d$ were obtained with the sample shown in Fig. 2a-d, respectively.) The curve $s$ expresses the decay kinetics obtained for PYP solution containing 45\% PEG 2000 and $100 \mathrm{mM}$ MES pH 6.5. The absorbance change of each sample was normalized by the absolute 
absorbance at $470 \mathrm{~nm}$ under dark, which makes the direct comparison of each curve possible. The vertical axis, thus, indicates the fraction of $\mathrm{PYP}_{\mathrm{M}}$ converted from dark-state with negative sign. It is clear that the initial amplitude at $t=0$ is different from sample to sample, indicating that the fraction converted to $\mathrm{PYP}_{\mathrm{M}}$ is different each other. The amplitude increased in the order from $a$ to $d$, in which is the reverse order of the crystallinity. Therefore, we conclude that the initial fraction of $\mathrm{PYP}_{\mathrm{M}}$ is closely correlated with the degree of crystallinity.

Each kinetics curve can be fit with a single exponential function to estimate the time constant for the decay of $\mathrm{PYP}_{\mathrm{M}}$ at each condition, except for curve $a$. The solid lines indicate the single exponential fits, which are superimposed on the experimental data shown as dot. The curve $a$ obtained from the sample (a) in Fig. 2 with large amount of crystal shows no time dependent changes. The initial amplitude for curve $a$ is also negligible small. These results suggests that the sample shown in Fig. 2a did not show any photoreaction.

Figure 5 shows the relative fraction of $\mathrm{PYP}_{\mathrm{M}}$ at $t=0$ to the amount of $\mathrm{PYP}_{\mathrm{M}}$ in solution with the rate constant estimated from the decay kinetics. The relative fraction of $\mathrm{PYP}_{\mathrm{M}}$ was increased with the decrease of crystallinity. On the other hand, the decay rates estimated from a single exponential fitting were kept constant among the polycrystalline samples obtained by gentle pressing and the solution sample. These results suggest that the $\mathrm{PYP}_{\mathrm{M}}$ observed here was converted from PYP in solution portion of the observed region, not in crystalline portions. In fact, the sample (a) containing a large amount of crystals shows no absorbance changes (Fig. 4a).

The results suggest that the crystalline condition influences the photoreaction of PYP. We examined the effect of hydration level of the sample to understand how crystalline condition affects the photoreaction. To estimate the hydration level in PYP crystal, the infrared absorption spectrum of PYP crystal was measured by an Infrared Microspectroscopy Station at BL43IR in SPring-8, and compared with that of the hydrated film. The crystal sample was prepared in the same way as the visible spectroscopic study. The used pressed crystal in FTIR measurements was checked by microscope equipped with polarizer. The pressed polycrystalline sample emitted green light with comparable intensity to the sample $a$ in Fig. 2, indicating the high crystallinity. The observed IR spectrum is shown in Fig. 6. The C-H stretching mode $\left(\sim 2900 \mathrm{~cm}^{-1}\right)$ for the crystal was more prominent than that for the hydrated film. The relatively stronger $\mathrm{C}-\mathrm{H}$ stretching mode than $\mathrm{O}-\mathrm{H}$ stretching mode $\left(\sim 3400 \mathrm{~cm}^{-1}\right)$ suggests that the content of water molecule in the crystal is lower than that in the hydrated film.

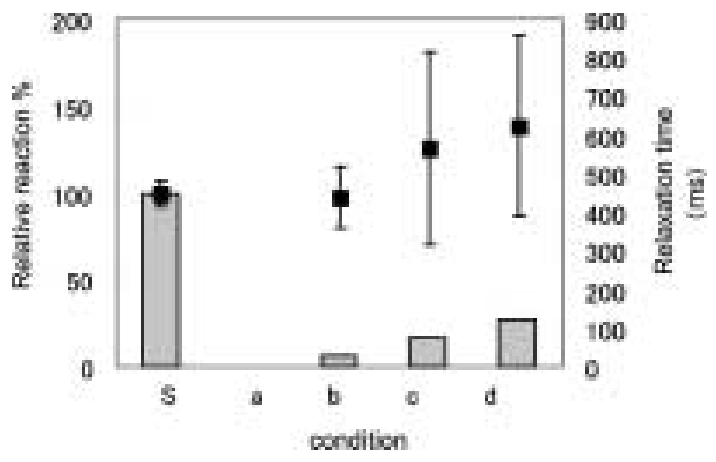

Fig. 5. The initial amplitude (bar) and relaxation time ( $\mathbf{\square}$ ) obtain by Fig. 4. The initial amplitude is normalized to the values for solution sample, 1, 2, 3, and 4 denotes the state a, b, c, and d of Fig. 2, respectively. S denotes solution condition. 


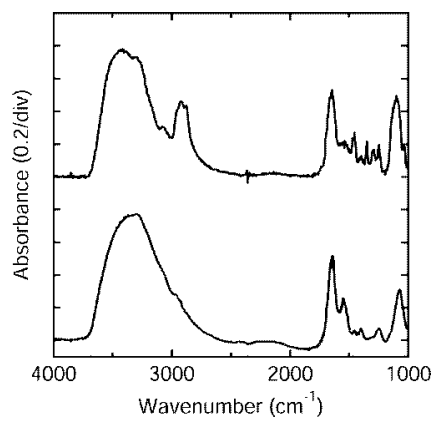

Fig. 6. The infrared absorption spectra of PYP crystal (top) and hydrated PYP film (bottom).

Table 1

Comparison of lifetime of $\mathrm{PYP}_{\mathrm{M}}$ in crystal and in solution

\begin{tabular}{|c|c|}
\hline Relaxation time & Crystal condition \\
\hline $474.76 \pm 58.6 \mathrm{msec}$ & $\mathrm{P}_{5}$ crystal $^{*}$ \\
\hline $447.58 \pm 32.6 \mathrm{msec}$ & $45 \%$ PEG2000 pH 6.5 \\
\hline $100 \mathrm{msec}$ (slow) & $\mathrm{P}_{3}$ crystal $^{* *}$ \\
\hline \multicolumn{2}{|l|}{30 msec (fast) } \\
\hline $200 \mathrm{msec}$ & $50 \%$ saturated ammonium sulfate $\mathrm{pH} 7.0^{* *}$ \\
\hline
\end{tabular}

\section{Discussion}

Two types of PYP crystals have been reported: one is in a space group $\mathrm{P}_{3}$ grown up by vapor diffusion against about $60 \%$ saturated ammonium sulfate at $\mathrm{pH} 7$ [19] and the other is in a space group $\mathrm{P}_{5}$ against 45\% PEG2000 pH 6.5 [16]. The former crystal was firstly obtained historically and provided the structure of dark state. The time-resolved X-ray crystallographic studies to determine the intermediate structure were also carried out using the $\mathrm{P}_{3}$ crystal [20]. The solved intermediate structure, corresponding to $\mathrm{PYP}_{\mathrm{M}}$, shows the structural changes only around the vicinity of the isomerized chromophore. The time-resolved absorption spectroscopic studies for PYP crystal in $\mathrm{P}_{3}$ have been reported to investigate the effect of crystal packing, and indicated that the photoreaction was highly influenced by the crystal packing (shown in the table) [21].

When we compare the two types of crystals, it is clear that the local contact with symmetry-related molecules in space group $\mathrm{P}_{5}$ is less than that in space group $\mathrm{P}_{3}$, suggesting that the molecular packing of $\mathrm{P} 6_{5}$ crystal was looser than that of $\mathrm{P}_{3}$ crystal. The loosen packing would be favorable to conformation rearrangement. In this paper, the photoreaction of the PYP crystal in space group P6 5 was examined. Our measurements showed that the absorbance change of $\mathrm{P} 6_{5}$ crystal after excitation was able to fit by a single exponential. However the calculated lifetime was close to that in 45\% PEG2000 solution (Table 1). The initial amplitude is smaller for the higher degree of crystallinity. These results suggest that only the PYP in solution contributes the observed decay kinetics. According to the previous study [22], the decay process of $\mathrm{P}_{3}$ crystal was composed of two components with different kinetics. Although the slow component has the comparable lifetime to that in 50\% saturated ammonium sulfate, the decay rate of fast component was 3 times faster than that in solution (Table 1). They concluded that the different reaction path from solution condition was realized in crystal condition. From the present result, we would like 
to point out the possibility that the slow component comes from PYP in solution, but not in crystal. In our experiment, the species corresponding to the fast component in $\mathrm{P}_{3}$ was not observed. The plausible explanation is that the $\mathrm{PYP}_{\mathrm{M}}$ is not formed upon illumination in $\mathrm{P}_{5}$ crystal and/or the decay rate is so accelerated that the decay kinetics cannot be observed with the time resolution of our experiment $(100 \mathrm{msec})$. Thus, we can conclude that the photoreaction of P6 6 crystal is also highly affected by the crystalline packing, even though it is looser than $\mathrm{P}_{3}$ crystal.

The difference FTIR spectrum between $\mathrm{PYP}_{\mathrm{M}}$ and dark-state was previously reported using hydrated film [18] and solution [14]. Major difference between the hydrated film and solution was observed around amide region. The absorbance change in the hydrated film was less prominent than in solution condition, suggesting that unsufficient water would inhibit the global conformational changes. The present FTIR measurements clarified that the water content of crystal sample was much less than that in hydrated film. Therefore, the effect on photoreaction under crystal condition would be derived not only from the crystal packing but also from the unsufficient water content in crystal condition.

In this study, the luminescence from crystal was used as the index of crystallinity. In the dissolved crystal, luminescence could not be observed. We have no definite information on the origin of the luminescence. However, if the luminescence was due to the fluorescence from crystal, the initial phase immediately after absorbing a photon, that is, the potential surface of excited state, might be also highly affected by the crystalline condition. To confirm the origin of luminescence, further study should be required.

These results lead to the conclusion that the crystal condition both in space group $\mathrm{P}_{3}$ and $\mathrm{P}_{5}$ considerably modify the properties of PYP, caused by the crystal packing and the unsufficient content of water. In order to interpret the molecular mechanism of PYP based on the intermediate crystal structure reported so far, it is necessary to characterize the difference in molecular events between in crystal condition and in solution condition precisely. We should keep the application limit of crystal structure in mind.

\section{Acknowledgements}

The infrared microspectroscopy at BL43IR at SPring-8 was performed under the approval of the Japan Synchrotron Radiation Research Institute (JASRI) (Proposal No. 2001B0360-NLS-np). We also thank Dr. Yuka Ikemoto of JASRI for helping us to set up the beamline for infrared microspectroscopy.

\section{References}

[1] T.E. Meyer, Biochim. Biophys. Acta 806 (1985), 175-183.

[2] J.J. Van Beeumen, B.V. Devreese, S.M. Van Bun, W.D. Hoff, K.J. Hellingwerf, T.E. Meyer, D.E. McRee and M.A. Cusanovich, Protein Sci. 2 (1993), 1114-1125.

[3] M. Baca, G.E.O. Borgstahl, M. Boissinot, P.M. Burke, D.R. Williams, K.A. Slater and E.D. Getzoff, Biochemistry 33 (1994), 14369-14 377.

[4] R. Kort, H. Vonk, X. Xu, W.D. Hoff, W. Crielaard and K.J. Hellingwerf, Biochemistry 34 (1996), 73-78.

[5] T.E. Meyer, E. Yakali, M.A. Cusanovich and G. Tollin, Biochemistry 26 (1987), 418-423.

[6] T.E. Meyer, G. Tollin, J.H. Hazzard and M.A. Cusanovich, Biophys. J. 56 (1989), 559-564.

[7] T.E. Meyer, G. Tollin, T.P. Causgrove, P. Cheng and R.E. Blankenship, Biophys. J. 59 (1991), 988-991.

[8] U.K. Genick, S.M. Soltis, P. Kuhn, I.L. Canestrelli and E.D. Getzoff, Nature 392 (1998), 206-209.

[9] B. Perman, V. Srajer, Z. Ren, T. Teng, C. Pradervand, T. Ursby, D. Bourgeois, F. Schotte, M. Wulff, R. Kort, K. Hellingwerf and K. Moffat, Science 279 (1998), 1946-1950.

[10] H. Kandori, T. Iwata, J. Hendriks, A. Maeda and K.J. Hellingwerf, Biochemistry 39 (2000), 7902-7909. 
[11] U.K. Genick, G.E. Borgstahl, K. Ng, Z. Ren, C. Pradervand, P.M. Burke, V. Srajer, T.Y. Teng, W. Schildkamp, D.E. McRee, K. Moffat and E.D. Getzoff, Science 275 (1997), 1471-1475.

[12] G. Rubinstenn, G.W. Vuister, F.A.A. Mulder, P.E. Dux, R. Boelens, K.J. Hellingwerf and R. Kamtein(Kaptein?), Nature Struct. Biol. 3 (1998), 568-570.

[13] N. Shimizu, H. Kamikubo, K. Mihara, Y. Imamoto and M. Kataoka, J. Biochem. 132 (2002), 257-263.

[14] A. Xie, L. Kelemen, J. Hendriks, B.J. White, K.J. Hellingwerf and W.D. Hoff, Biochemistry 40 (2001), 1510-1517.

[15] Y. Imamoto, T. Ito, M. Kataoka and F. Tokunaga, FEBS Lett. 374 (1995), 157-160.

[16] K. Mihara, O. Hisatomi, Y. Imamoto, M. Kataoka and F. Tokunaga, J. Biochem. 121 (1997), 876-880.

[17] D.M.F. van Aalten, W. Crielaard, K.J. Hellingwerf and L. Joshua-Tor, Protein Sci. 9 (2000), 64-72.

[18] Y. Imamoto, Y. Shirahige, F. Tokunaga, T. Kinoshita, K. Yoshihara and M. Kataoka, Biochemistry 40 (2001), 8997-9004.

[19] M. Harigai, S. Yasuda, Y. Imamoto, K. Yoshihara, F. Tokunaga and M. Kataoka, J. Biochem. 130 (2001), 51-56.

[20] B. Perman, V. Srajer, Z. Ren, T. Teng, C. Pradervand, T. Ursby, D. Bourgeois, F. Schotte, M. Wulff, K. Hellingwerf and K. Moffat, Science 279 (1998), 1946-1950.

[21] D.E. McRee, T.E. Meyer, M.A. Cusanovich, H.E. Parge and E.D. Getzoff, J. Biol. Chem. 261 (1986), 13 850-13 851.

[22] K. Ng, E.D. Getzoff and K. Moffat, Biochemistry 34 (1995), 879-890. 


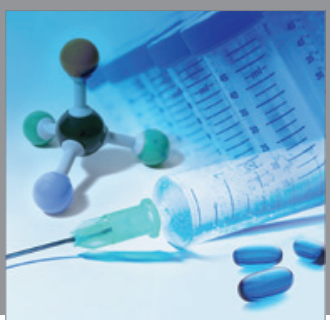

International Journal of

Medicinal Chemistry

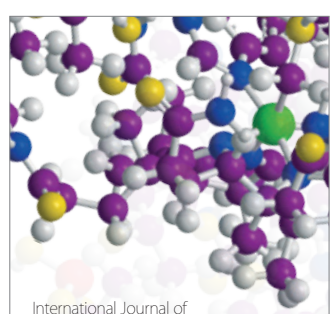

Carbohydrate Chemistry

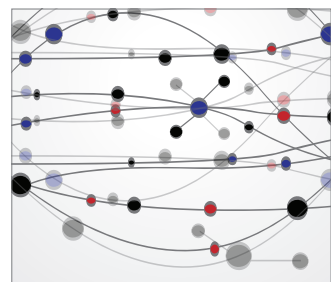

The Scientific World Journal
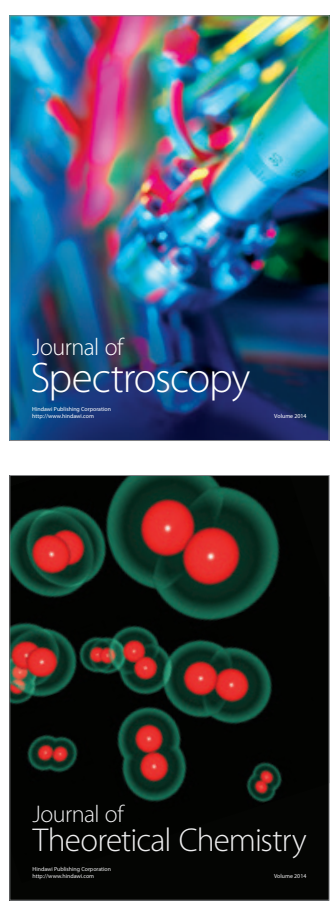
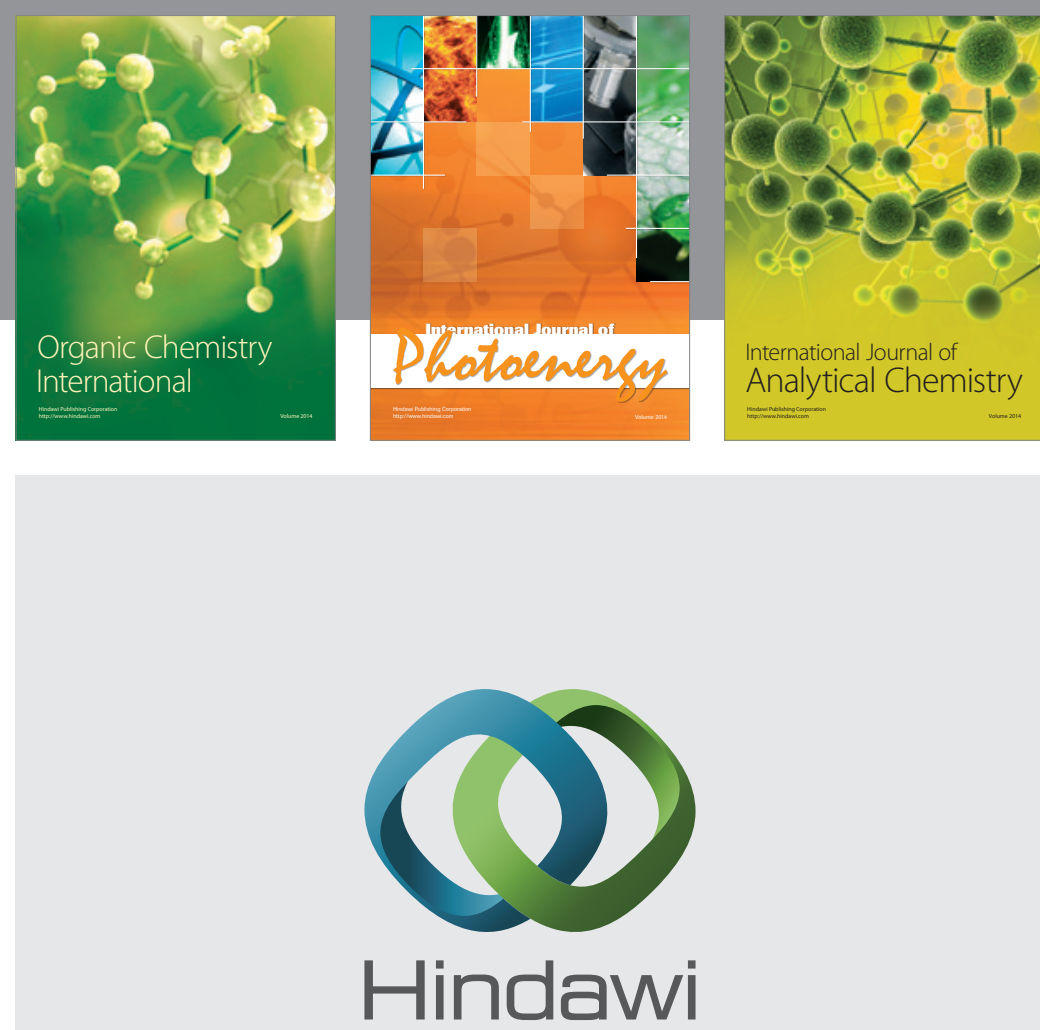

Submit your manuscripts at

http://www.hindawi.com
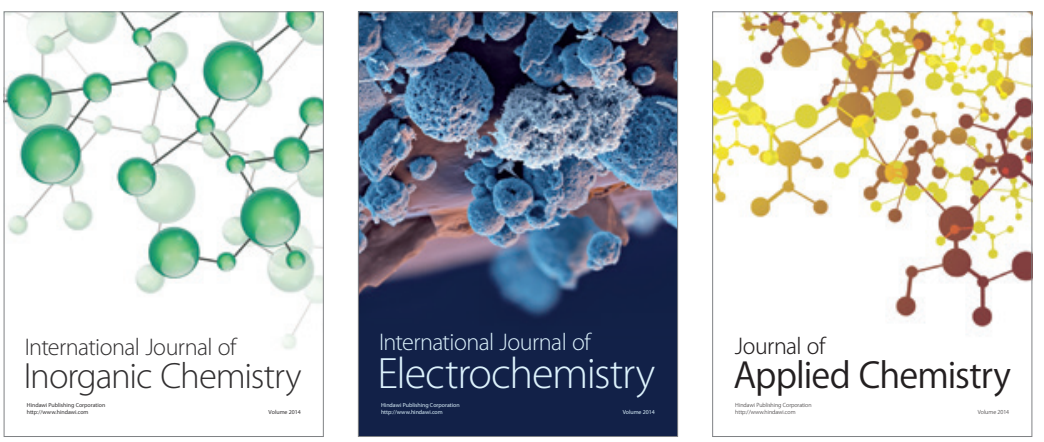

Journal of

Applied Chemistry
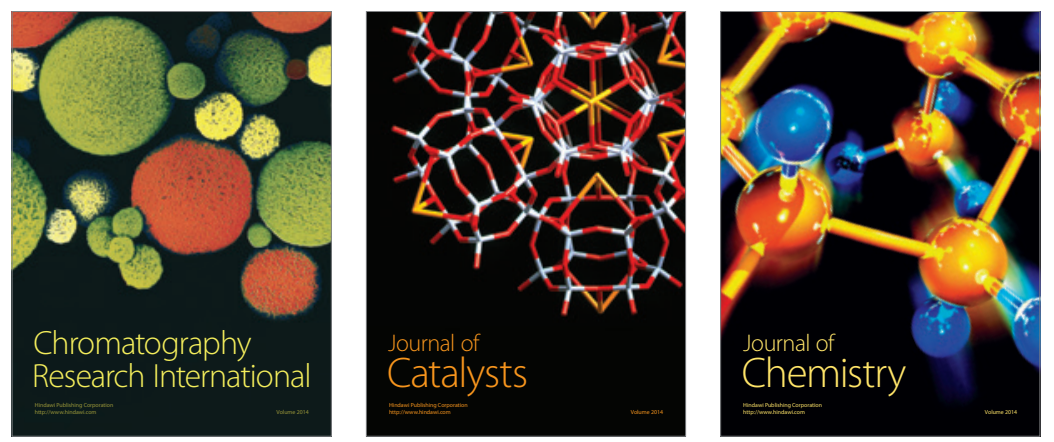
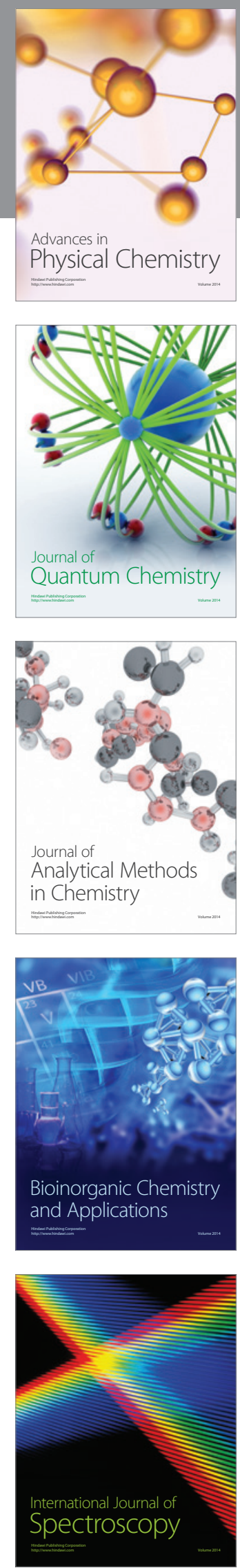